Jürgen Jost

\title{
Riemannian Geometry \\ and Geometric Analysis
}

Fifth Edition

글 


\section{Contents}

1 Foundational Material 1

1.1 Manifolds and Differentiable Manifolds . . . . . . . . . . . . . . 1

1.2 Tangent Spaces . . . . . . . . . . . . . . . . . . 6

1.3 Submanifolds . . . . . . . . . . . . . . . . . . . 10

1.4 Riemannian Metrics . . . . . . . . . . . . . . . . . . . 13

1.5 Existence of Geodesics on Compact Manifolds . . . . . . . . . . . 28

1.6 The Heat Flow and the Existence of Geodesics . . . . . . . . . . . 31

1.7 Existence of Geodesics on Complete Manifolds . . . . . . . . . . . 34

1.8 Vector Bundles . . . . . . . . . . . . . . . . . . . . 37

1.9 Integral Curves of Vector Fields. Lie Algebras . . . . . . . . . . 47

1.10 Lie Groups . . . . . . . . . . . . . . . . . . . 56

1.11 Spin Structures . . . . . . . . . . . . . . . . 62

Exercises for Chapter $1 \ldots \ldots \ldots \ldots \ldots$. . . . . . . . 83

2 De Rham Cohomology and Harmonic Differential Forms $\quad 87$

2.1 The Laplace Operator . . . . . . . . . . . . . . . . . . . . . . . . 87

2.2 Representing Cohomology Classes by Harmonic Forms . . . . . . . 96

2.3 Generalizations . . . . . . . . . . . . . . . . . . . . . . . 104

2.4 The Heat Flow and Harmonic Forms . . . . . . . . . . . . 105

Exercises for Chapter $2 \ldots \ldots \ldots \ldots \ldots$

3 Parallel Transport, Connections, and Covariant Derivatives 113

3.1 Connections in Vector Bundles . . . . . . . . . . . . . . . . 113

3.2 Metric Connections. The Yang-Mills Functional . . . . . . . . . . . . 124

3.3 The Levi-Civita Connection . . . . . . . . . . . . . . . . . . . . . 140

3.4 Connections for Spin Structures and the Dirac Operator . . . . . . . 155

3.5 The Bochner Method . . . . . . . . . . . . . . . . . . . . 162

3.6 The Geometry of Submanifolds. Minimal Submanifolds . . . . . . . 164

Exercises for Chapter $3 \ldots \ldots \ldots \ldots \ldots$

4 Geodesics and Jacobi Fields 179

4.1 1st and 2nd Variation of Arc Length and Energy . . . . . . . . 179

4.2 Jacobi Fields . . . . . . . . . . . . . . . . . . . . . . 185 
4.3 Conjugate Points and Distance Minimizing Geodesics _ . . . . . . 193

4.4 Riemannian Manifolds of Constant Curvature . . . . . . . . . . . . . 201

4.5 The Rauch Comparison Theorems and Other Jacobi Field Estimates . 203

4.6 Geometric Applications of Jacobi Field Estimates . . . . . . . . . . . 208

4.7 Approximate Fundamental Solutions and Representation Formulae . . 213

4.8 The Geometry of Manifolds of Nonpositive Sectional Curvature . . . . 215

Exercises for Chapter $4 \ldots \ldots \ldots$. . . . . . . . . . . 232

A Short Survey on Curvature and Topology 235

5 Symmetric Spaces and Kähler Manifolds 243

5.1 Complex Projective Space . . . . . . . . . . . . . . . . . . 243

5.2 Kähler Manifolds . . . . . . . . . . . . . . . . . . . . . . . . . . . 249

5.3 The Geometry of Symmetric Spaces . . . . . . . . . . . . . . . 259

5.4 Some Results about the Structure of Symmetric Spaces . . . . . . . 270

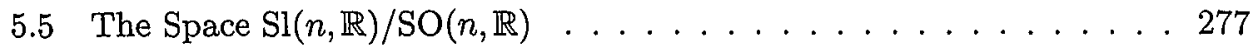

5.6 Symmetric Spaces of Noncompact Type . . . . . . . . . . . . . 294

Exercises for Chapter 5 . . . . . . . . . . . . . . . . . . . . 299

6 Morse Theory and Floer Homology 301

6.1 Preliminaries: Aims of Morse Theory . . . . . . . . . . . . . . . . . . 301

6.2 The Palais-Smale Condition, Existence of Saddle Points . . . . . . 306

6.3 Local Analysis . . . . . . . . . . . . . . . . . . . . . . . . 308

6.4 Limits of Trajectories of the Gradient Flow . . . . . . . . . . . . 324

6.5 Floer Condition, Transversality and $\mathbb{Z}_{2}$-Cohomology . . . . . . . 332

6.6 Orientations and $\mathbb{Z}$-homology . . . . . . . . . . . . . . . 338

6.7 Homotopies . . . . . . . . . . . . . . . . . . . . . . . . . . 342

6.8 Graph flows . . . . . . . . . . . . . . . . . . . . . 346

6.9 Orientations . . . . . . . . . . . . . . . . . . . . 350

6.10 The Morse Inequalities . . . . . . . . . . . . . . . . . . . . . 366

6.11 The Palais-Smale Condition and the Existence of Closed Geodesics . . 377

Exercises for Chapter $6 \ldots \ldots$. . . . . . . . . . . . 390

7 Harmonic Maps between Riemannian Manifolds 393

7.1 Definitions . . . . . . . . . . . . . . . . . . . . . . . 393

7.2 Formulae for Harmonic Maps. The Bochner Technique . . . . . . . . 400

7.3 The Energy Integral and Weakly Harmonic Maps . . . . . . . . . . . 412

7.4 Higher Regularity . . . . . . . . . . . . . . . . . . . . . . . 422

7.5 Existence of Harmonic Maps for Nonpositive Curvature . . . . . . . . 433

7.6 Regularity of Harmonic Maps for Nonpositive Curvature . . . . . . . . 440

7.7 Harmonic Map Uniqueness and Applications . . . . . . . . . . . . . . 459

Exercises for Chapter $7 \ldots \ldots \ldots \ldots$. . . . . . . . . 466

8 Harmonic maps from Riemann surfaces $\quad 469$

8.1 Twodimensional Harmonic Mappings . . . . . . . . . . . . . . . . . . 469

8.2 The Existence of Harmonic Maps in Two Dimensions . . . . . . . . . . 483 
8.3 Regularity Results . . . . . . . . . . . . . . . . . . . . . 504

Exercises for Chapter $8 \ldots \ldots \ldots \ldots \ldots$. . . . . . . . . . . . . . . . .

9 Variational Problems from Quantum Field Theory 521

9.1 The Ginzburg-Landau Functional . . . . . . . . . . . . . . . . . . . . 521

9.2 The Seiberg-Witten Functional . . . . . . . . . . . . . . . . 529

9.3 Dirac-harmonic Maps . . . . . . . . . . . . . . . . . . . . . 536

Exercises for Chapter 9 . . . . . . . . . . . . . . . . . . . . . 543

A Linear Elliptic Partial Differential Equations 545

A.1 Sobolev Spaces . . . . . . . . . . . . . . . . . . . . . . 545

A.2 Linear Elliptic Equations . . . . . . . . . . . . . . . . . . . 549

A.3 Linear Parabolic Equations . . . . . . . . . . . . . . . 553

B Fundamental Groups and Covering Spaces

$\begin{array}{ll}\text { Bibliography } & \mathbf{5 6 0}\end{array}$

Index $\quad 576$ 